\title{
Whole-body magnetic resonance imaging (WBMRI) versus whole-body computed tomography (WBCT) for myeloma imaging and staging
}

\author{
Karla M. Treitl $^{1}$ (D) Jens Ricke ${ }^{1} \cdot$ Andrea Baur-Melnyk $^{1}$ \\ Received: 17 February 2021 / Revised: 19 April 2021 / Accepted: 25 April 2021 / Published online: 24 May 2021 \\ (C) The Author(s) 2021
}

\begin{abstract}
Myeloma-associated bone disease (MBD) develops in about $80-90 \%$ of patients and severely affects their quality of life, as it accounts for the majority of mortality and morbidity. Imaging in multiple myeloma (MM) and MBD is of utmost importance in order to detect bone and bone marrow lesions as well as extraosseous soft-tissue masses and complications before the initiation of treatment. It is required for determination of the stage of disease and aids in the assessment of treatment response. Whole-body low-dose computed tomography (WBLDCT) is the key modality to establish the initial diagnosis of MM and is now recommended as reference standard procedure for the detection of lytic destruction in MBD. In contrast, whole-body magnetic resonance imaging (WBMRI) has higher sensitivity for the detection of focal and diffuse plasma cell infiltration patterns of the bone marrow and identifies them prior to osteolytic destruction. It is recommended for the evaluation of spinal and vertebral lesions, while functional, diffusion-weighted MRI (DWI-MRI) is a promising tool for the assessment of treatment response. This review addresses the current improvements and limitations of WBCT and WBMRI for diagnosis and staging in MM, underlining the fact that both modalities offer complementary information. It further summarizes the corresponding radiological findings and novel technological aspects of both modalities.
\end{abstract}

Keywords Plasma cell disorders $\cdot$ Multiple myeloma $\cdot$ Computed tomography $\cdot$ Magnetic resonance imaging $\cdot$ Whole-body imaging

\section{Introduction}

Plasma cell disorders (PCD) are a heterogenous group of monoclonal malignancies that evolve from terminally differentiated plasma cells and are classified according to the International Myeloma Working Group (IMWG) criteria [1]. Multiple myeloma (MM) accounts for $1 \%$ of all cancers and $10 \%$ of all hematological diseases [2]. In patients suffering from $\mathrm{MM}$, the uncontrolled clonal proliferation of terminally differentiated B cells can be verified by bone marrow aspiration. It is associated with specific end-organ damage such as

Karla M. Treitl

Karlamaria.Treitl@med.uni-muenchen.de

1 Department of Radiology, University Hospital, LMU Munich, Marchioninistr. 15, 81377 Munich, Germany hypercalcemia, renal insufficiency, anemia or destructive bone lesions (CRAB criteria) [3, 4]. Alternatively, specific biomarkers, including a serum free light-chain ratio $\geq 100$ or $\geq$ $60 \%$ of plasma cells in the bone marrow, indicate active MM, as well as magnetic resonance imaging (MRI) scans that reveal more than one focal bone lesion at least $5 \mathrm{~mm}$ in diameter [5]. Thus, MR imaging is for the first time a specific biomarker for the diagnosis of MM.

Myeloma-associated bone disease (MBD) is the most frequent manifestation of MM and accounts for the majority of mortality and morbidity associated with this PCD. The pathophysiological cause is a dysregulation of osteoblastic and osteoclastic interactions, leading to increased bone resorption without compensating bone formation, due to suppressed osteoblastic activity and upregulated osteoclastic activity [5]. It is characterized by the presence of osteolytic bone lesions on computed tomography (CT) $[1,5,6]$. Actually, $90 \%$ of patients will develop destructive bone lesions, while $80 \%$ will even suffer from a pathological fracture during the 
progression of their disease [7-9]. Therefore, MBD imaging is essential in the diagnosis, prognosis and follow-up of MM.

Imaging can further confirm the transition of a preliminary or intermediate stage of PCD, so-called monoclonal gammopathy of undetermined significance (MGUS) or smoldering multiple myeloma (SMM), to active MM by detecting osteolytic destruction. Furthermore, it is essential to differentiate solitary plasmacytoma from $\mathrm{MM}$ by $\left[{ }^{18} \mathrm{~F}\right]$ fluorodeoxyglucose positron emission tomography-CT $\left(\left[{ }^{18} \mathrm{~F}\right]-\mathrm{FDG}\right.$ PET/CT) or MRI. MRI is the major diagnostic tool for identifying extraosseous manifestations and complications, such as pathological fractures and spinal cord compression.

The aim of this article is to review and compare wholebody CT (WBCT) and whole-body MRI (WBMRI) for diagnosis and staging in $\mathrm{MM}$ and to summarize the corresponding radiological findings.

\section{X-ray imaging (XR)}

Conventional radiography (XR) underestimates the stage of disease and misses myeloma-related bone lesions in up to $70 \%$ of cases when compared to cross-sectional imaging techniques, as lesions become detectable on XR only after a decrease of at least $30-50 \%$ of bone mineral density $[10,11]$. Therefore, cross-sectional and functional imaging techniques have replaced the whole-body radiographic skeletal (WBXR) survey as the gold standard for the radiological screening and diagnosis of MM. Both WBCT and WBMRI can diagnose intra- and extramedullary manifestations of MM with greater sensitivity and detect disease progression or relapse much earlier $[12,13]$. They further provide prognostic information and are essential for the evaluation of therapeutic efficacy during staging and follow-up examinations $[2,14]$.

\section{Whole-body computed tomography (WBCT)}

\section{Modality overview}

As a cross-sectional imaging technique, whole-body computed tomography (WBCT) provides three-dimensional information and is able to display bone abnormalities associated with MM. WBCT is much more sensitive for detecting osteolytic lesions than WBXR because of the high contrast between cortical and cancellous bone tissue [15]. Since 2014, the guidelines of the IMWG state that the detection of one or more osteolytic bone lesions at least $5 \mathrm{~mm}$ in size on CT, including whole-body low-dose CT (WBLDCT) or PET/CT, meets the criteria for MBD [6]. In consequence, according to the European Myeloma Network and the European Society for Medical Oncology and the IMWG, WBLDCT is recommended as the initial reference standard procedure and the modality of choice to detect osteolytic MBD $[16,17]$. Almost every MM patient receives a WBLDCT as baseline and follow-up staging examination [6]. $\left[{ }^{18} \mathrm{~F}\right]-\mathrm{FDG}$ PET/CT is a valuable alternative that is able to detect lytic bone lesions and extraosseous masses, while providing information based on the $\left[{ }^{18} \mathrm{~F}\right]-\mathrm{FDG}$ activity as well [18].

The IMWG guidelines further emphasize to need to avoid over-interpretation of small lucencies with a diameter below the threshold of $5 \mathrm{~mm}$ on CT images, and recommend reexamination of these particular patients after 3-6 months. Important differential diagnoses for hypodense trabecular bone lesions are benign fat-containing processes, which can be identified as false-positive lesions by $\mathrm{CT}$ density measurements. The most frequent examples are focal yellow bone marrow deposits, small hemangiomas and Modic type 2 degenerative vertebral endplate changes. Alternatively, an additional MRI examination or a biopsy of the specific lesions should be taken into consideration, if possible, in order to appropriately characterize them before establishing the diagnosis of MM and initiating systemic therapy. As MM is defined either by the percentage of bone marrow plasma cells $\geq 10 \%$ or biopsy-proven histological evidence of bony or extramedullary plasmacytoma, follow-up imaging is required if these criteria do not apply [14].

In patients with suspected SMM, the IMWG recommends at least one type of advanced imaging (PET/CT, WBLDCT, MRI of the pelvis and spine or WBMRI), depending on the availability and clinical situation, in order to distinguish SMM from active MM. Follow-up examinations are indicated in cases with changes in the clinical course or changing $\mathrm{M}$ protein levels. High-risk patients in particular should receive periodic staging imaging to exclude asymptomatic progression [19]. Although patients with SMM may remain stable over extended periods of time, the progression rate to active MM is reported as $10 \%$ per year for the first 5 years and $3 \%$ per year for the following 5 years $[20,21]$.

WBLDCT further reliably excludes osteolysis in patients with MGUS and thereby completes the diagnostic hematological results in these cases. It also proved to be very sensitive in the depiction of newly developed lytic lesions in follow-up examinations of patients suffering from MGUS, who have an overall $1 \%$ risk of developing active MM $[20,22]$. Risk factors for the progression of MGUS to active MM are M-protein levels of $1.5 \mathrm{~g} / \mathrm{dl}$ or more and an abnormal free light-chain ratio in patients with non-IgM MGUS. However, the progression rate differs considerably between patients with no, one or two risk factors, and ranges from $7 \%$ to $20 \%$ and even $30 \%$ over 20 years [23]. Therefore, the IMWG recommends WBLDCT only in patients with high-risk MGUS.

In addition, $\mathrm{CT}$ is necessary for the planning of surgery in order to evaluate bony structure. Patients with osteoporosis need cement-augmented screw fixations, as osteolysis in the 
pedicles prohibits pedicle screws in this location. Another benefit of CT is its assistance in percutaneous biopsy for suspected lesions, in particular as the plasma cell percentage differs significantly between random bone marrow biopsy specimens and CT-guided samples, especially in focal disease $[24,25]$. This in turn influences the final diagnosis, the staging of the disease and consequent treatment strategy.

\section{Imaging findings in WBLDCT}

Lytic, well-circumscribed areas with non-sclerotic margins, producing a "punched-out" aspect, are regarded as typical and the most common manifestation of osteolytic lesions in MBD. The non-sclerotic halo is in contrast to most other bone metastases and indicates the decrease in osteoblastic activity (Fig. 1). They are primarily located in the trabecular bone of the axial skeleton and the pelvis, less frequently in the long bones of the upper and lower extremities [8, 14]. On WBLDCT, they are easily distinguished against normally mineralized trabecular bone, but may be difficult to distinguish in patients with advanced osteoporosis and rarefied trabeculae. Fat-containing, hypodense, benign lesions of the trabecular bone, such as typical hemangiomas, need to be discriminated from osteolytic lesions by density measurements. However, the detection of fat excludes osteolysis only in untreated patients, because treated lesions may feature a partial or total fatty replacement [17].

WBLDCT can further identify focal plasma cell infiltrates within the bone marrow of the appendicular skeleton, where they can be discriminated against fatty hypodense marrow $(-30$

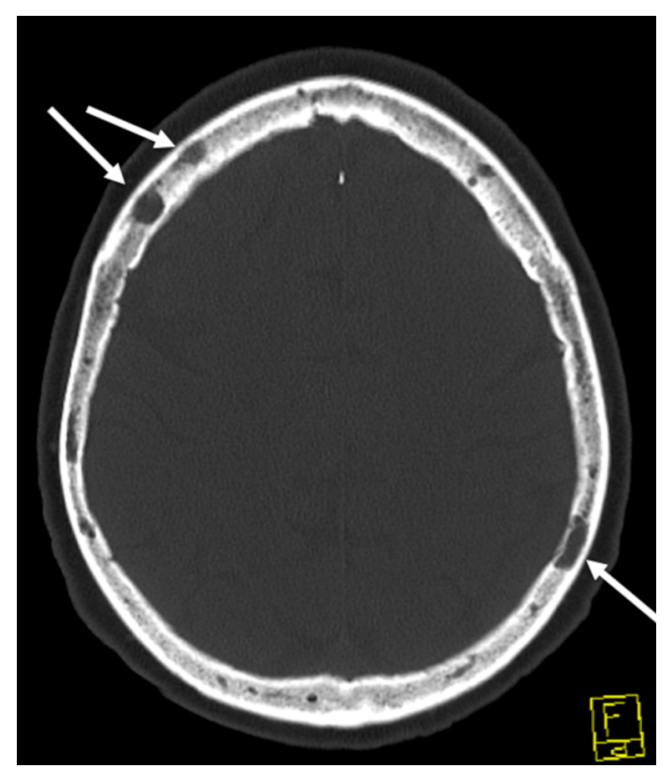

Fig. 1 Axial view of a CT scan of the head in the bone window. The white arrows mark typical punched-out osteolytic lesions in the skull of a 48-year-old female patient with multiple myeloma
$\mathrm{HU}$ to $-100 \mathrm{HU}$ ), due to a higher density (average $55 \mathrm{HU}$ ) [14, 26]. A prospective study including 172 patients suffering from MGUS, SMM or active MM revealed that the percentage of medullary abnormalities and their CT density increased with increasing stage of disease. The authors consequently concluded that the CT density of focal medullary lesions of the appendicular skeleton correlates with disease progression and has prognostic value for the course of the disease [27].

Two different patterns of lytic bone lesions in MM were recently identified on WBLDCT [28]. The first pattern, with negative CT density values, had low ${ }^{18}[\mathrm{~F}]$-FDG-uptake on $\mathrm{PET} / \mathrm{CT}$ and a low apparent diffusion coefficient (ADC) on MRI, which is consistent with fatty marrow. The second pattern, with positive CT density values, had higher FDG-uptake on PET/CT and a higher ADC value on MRI, which is consistent with an infiltrative pattern. However, histology documented a plasma cell infiltration in both types of lesions and revealed that neoplastic plasma cells were scattered among adipocytes in the first pattern. Moreover, both patterns were simultaneously found in patients with symptomatic bone disease indicating active MM. Additionally, some of the HUnegative lesions became HU-positive in follow-up WBLDCT and developed an FDG-uptake and a pathological ADC. The investigators concluded that the initially hypodense, fat-rich lesions represent a preliminary stage that advances into hyperdense, infiltrative lesions with a higher concentration of plasma cells. However, the current IMWG criteria do not incorporate the $\mathrm{CT}$ density of myeloma lesions.

Endosteal scalloping and cortical disruption are typical findings when osteolytic lesions extend into the cortex of bones. When the cortex is disrupted, they can show softtissue masses. In some rare cases, MM may present osteosclerotic lesions, especially in POEMS (polyneuropathy, organomegaly, endocrinopathy, monoclonal protein, skin changes) syndrome, which is addressed in a separate article in this special issue.

A diffuse infiltration pattern in MM presents as diffuse osteoporosis and is hard to discriminate from senile or postmenopausal osteoporosis on WBLDCT. Age-related osteoporosis is associated with increasing fat content of the bone marrow, while a diffuse infiltration causes hypercellularity. MRI is the modality of choice to depict the hypercellularity in these cases using enhanced and unenhanced T1-weighted (T1w) spin-echo (SE) sequences [29, 30]. Dual-energy CT (DECT) may overcome this problem by generating virtual non-calcium (VNCa) images. However, the technique is not yet available everywhere.

In addition to bone imaging, WBLDCT is able to identify extraosseous or extramedullary myeloma, which can either appear as para-osseous soft-tissue masses adjacent to bones or may atypically manifest as pulmonary, intra-abdominal or intracranial tumoral lesions. The most frequently involved anatomical structures are the head and neck region, lymph 
nodes, the pleura, the spleen and the liver [20, 31, 32]. The sensitivity for the detection of extraosseous myeloma on CT images is increased by the intravenous administration of contrast. The presence of extraosseous disease and an extramedullary relapse of MM are associated with a poorer prognosis and decreased survival rates, with few remaining therapeutic options. Their cross-sectional dimension and the assessment of growth or shrinkage with either technique are two aspects included in the standard IMWG response criteria defining a partial response and a minimal response [13]. FDGPET-CT has alternatively become established for the evaluation of extraosseous disease, since it is more sensitive than CT due to the uptake of FDG in these lesions and because it yields morphological, biochemical and functional information [18, $25,33]$.

Skeletal-related complications in MBD negatively affect survival [34]. Diffuse osteopenia and vertebral compression fractures, which are observed in up to $80 \%$ of patients, are typical complications of MM but are no longer considered diagnostic disease criteria [21, 35]. This is due mainly to the fact that it is sometimes difficult to establish the exact cause of the fracture by CT. As a quick, affordable, easily accessible and nearly ubiquitously available imaging technique, WBCT is the modality of choice to assess spinal stability in cases with pathological neoplastic or osteoporotic fractures and in acute trauma or staging examinations. This assessment via $\mathrm{CT}$ is crucial, especially since $11-24 \%$ of patients with MM experience spinal cord compression during the course of their disease.

\section{Radiation dose in WBLDCT}

Because of the high cumulative radiation dose from standard CT scans, WBLDCT has become the modality of choice for staging. Currently, the effective radiation dose is dependent on the type of CT scanner, and results from either lower kilovoltage peak $(\mathrm{kVp})$ or lower tube current-time product (mAs) or a combination of both parameters. In 2008 we performed the first comparative studies on WBLDCT and WBMRI, where we used a multidetector CT scanner with 16 or 64 detector rows, a constant tube voltage of $120 \mathrm{kV}$ and a tube current-time product of $100 \mathrm{mAs}$ [11]. In 2005, Horger et al. presented a scan protocol calculated at a tube currenttime product of $40 \mathrm{mAs}$ with an effective radiation dose of a $4.1 \mathrm{mSv}$ and $120 \mathrm{kV}$ that was only 1.7 -fold higher than the average radiation dose of XR (2.4 mSv) [36]. Technical advancements including automated modulation of the tube current and novel reconstruction algorithms have further improved the image quality and simultaneously reduced the effective radiation dose [37, 38].

A recent study examining 74 patients with various plasma cell diseases revealed that iterative reconstruction algorithms enabled WBLDCT scans with almost identical radiation exposure ( $2.7 \pm 0,9$ vs. $2.5 \pm 0,9 \mathrm{mSv} ; P=0,054)$, but superior detectability of bone lesions, skeletal fractures and vertebral compressions, in comparison to WBXR [39]. In 2018, the IMWG bone working group recommended the application of iterative reconstruction algorithms, if available, in order to reduce the image noise and streak artifacts of the WBLDCT images [17].

Dose reduction while maintaining the same image quality can alternatively be achieved by spectral shaping in WBLDCT [40]. Supplemental to the standard aluminum bowtie filter, spectral shaping uses an additional tin filter between the CT tube and the patient in order to absorb lowenergy photons, which are less pertinent in high-contrast imaging of the lung or the bones. The corresponding retrospective case-control study compared the WB scan protocols of a third- and second-generation dual-source $\mathrm{CT}$ scanner at $100 \mathrm{kV}$ in a total of 60 individuals, and resulted in a reduction of the mean effective dose of $74 \%$. A recently published study investigating 30 patients using spectral shaping and a thirdgeneration dual-source multidetector CT scanner further improved the effective dose [41]. Other improvements in dose reduction may be achieved by deep convolutional neural networks that are trained to remove CT image noise in order to improve the image quality of ultralow-dose $\mathrm{CT}$ scans to appear similar to normal-dose CT scans [42]. A 3D convolutional neural network was recently introduced with specific loss functions for QCT noise reduction to compute microstructural parameters such as tissue mineral density and bone volume ratio. This method allows the assessment of the $3 \mathrm{D}$ bone microstructure and has the potential to detect rarefaction of the trabecular network due to osteoporosis or other bone diseases [43]. It may further discriminate patients with and without vertebral fractures or indicate early stages of osteolytic processes that are not yet visible [44].

After all, further dose reduction of WBLDCT is preferable either way, considering the increasing relevance of surveillance imaging in patients suffering from MGUS and SMM.

Another technical achievement is DECT. Three different studies have shown that these calculated VNCa images allow the identification of plasma cell infiltration via color-coded maps and enable their quantification using region of interest (ROI)-based HU measurements [45-47]. A separate article in this issue addresses this topic.

\section{Positioning and technical parameters of WBLDCT}

As described above, there are several options for producing $\mathrm{CT}$ images of reasonable diagnostic quality while maintaining acceptable radiation doses. In 2018, the IMWG bone working group published specific recommendations for the acquisition of WBLDCT in MM [17, 25].

No specific patient preparation and no oral or intravenous contrast administration is required for the acquisition of bone 
images. The patient lies supine on the CT table, and in order to enable precise evaluation, the entire skull, both humeri and both proximal tibia metaphyses should be included in the field of view. Therefore, both arms can be placed aside and above the head, with as little bending of the elbows as possible in order to avoid truncation artifacts. Alternatively, they can be put alongside the body, but underlaid with custom-made arm cushions that lift them above the level of the thoracic and lumbar spine in order to avoid beam-hardening artifacts. The arms of obese patients may also be placed in front of the body with both hands folded in order to maintain an elevated position and to shift the beam-hardening artifacts anteriorly [17].

A multidetector CT scanner with at least 16 detector rows is required for WBLDCT in MM to ensure short scan times and to acquire thin slices while maintaining low cumulative radiation doses. As there are various data but no validated evidence for the optimal combination of tube voltage and tube current-time product, the IMWG bone working group recommends the application of $120 \mathrm{kV}$ combined with 50-70 mAs and a collimation range of $0.5-1.5 \mathrm{~mm}$. Image reconstruction should utilize a sharp, high-spatial-frequency convolution kernel to ensure increased spatial resolution enabling an optimal assessment of even small osteolytic lesions. Additional image reconstruction using a smooth convolution kernel ensures the optimal assessment of para- and extramedullary soft-tissue structures and enables the detection and attenuation measurement of medullary plasma cell infiltrates in the appendicular skeleton. Thin axial slices with an increment of $50 \%$ enable the detection of small osteolytic lesions and may serve as source images for multiplanar reformations (Fig. 2). Table 1 summarizes the abovementioned recommended technical parameters and includes accepted alternatives. As a matter of course, periodic calibration of the CT scanners ensures uniformity of the attenuation measurements over a period of time.

\section{Whole-body magnetic resonance imaging (WBMRI)}

\section{Modality overview}

The major advantage of MRI in comparison to other crosssectional imaging techniques is its superior soft-tissue contrast, offering the opportunity to visualize bone marrow

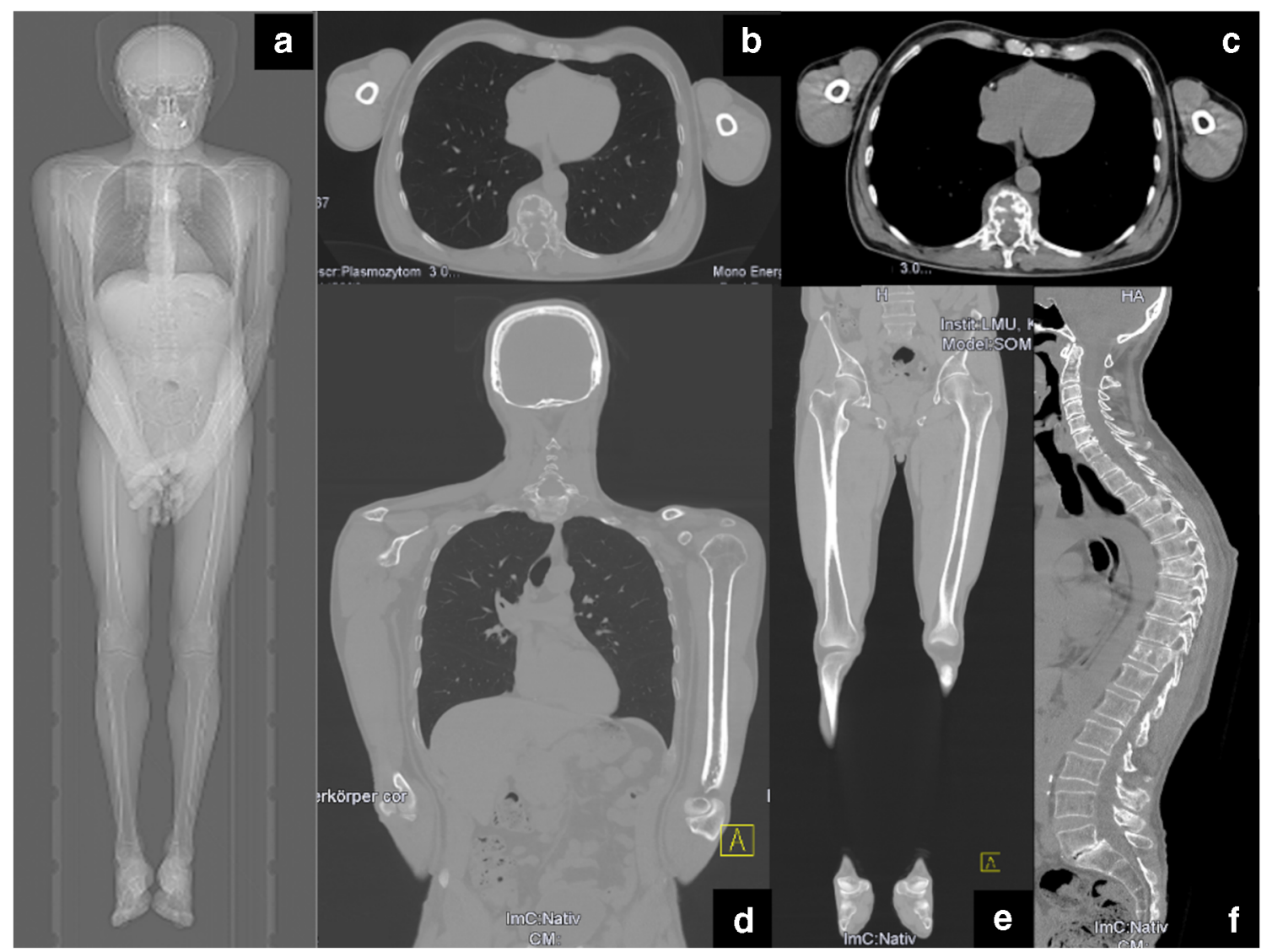

Fig. 2 Image examples of a whole-body low-dose CT scan (a: scout). Images were reconstructed in axial $3 \mathrm{~mm}$ slices using the intermediate (b) and soft-tissue kernel (c) in order to evaluate soft-tissue masses and the intrathoracic and intra-abdominal organs. The skeleton is further reconstructed in coronal $3 \mathrm{~mm}$ slices using the bone kernel for the reading of the upper (skull, thorax, arms; $\mathbf{b}, d$ ) and the lower parts (pelvis, legs; e) of the body. The spine is additionally reconstructed in sagittal orientation (f). 
Table 1 Summary of the recommended technical parameters and accepted alternatives for WBLDCT in MM [17]

\begin{tabular}{|c|c|c|}
\hline Parameters & Recommendation & Alternative \\
\hline No. of detector rows & At least 16 & \\
\hline Field of view & $\begin{array}{l}\text { From the cranial vertex to the prox. metaphyses } \\
\text { of both tibiae incl. both humeri }\end{array}$ & \\
\hline Regular patient positioning & $\begin{array}{l}\text { Supine with both arms aside and above the skull } \\
\text { with as little bending of the elbows as possible }\end{array}$ & $\begin{array}{l}\text { Supine with both arms alongside the body } \\
\text { underlaid with armrests to lift them above spine level }\end{array}$ \\
\hline Positioning of obese or PRM & $\begin{array}{l}\text { Supine with both arms placed in front of the body } \\
\text { with both hands folded }\end{array}$ & \\
\hline Tube voltage $(\mathrm{kV})$ & 120 & 140 \\
\hline Tube current-time product (mAs) & $50-70$ & $14-25$ \\
\hline Collimation & $0.5-1.5 \mathrm{~mm}$ & - \\
\hline Reconstruction convolution kernel & $\begin{array}{l}\text { Combination of a sharp, high-frequency "bone" } \\
\text { kernel and a smoother "soft-tissue" kernel }\end{array}$ & $\begin{array}{l}\text { One singular intermediate, middle-frequency } \\
\text { kernel permitting acquisition of all images } \\
\text { with different window settings* }\end{array}$ \\
\hline Iterative reconstruction algorithms & $\begin{array}{l}\text { Requested, if possible, to reduce image noise and } \\
\text { artifacts while reducing radiation exposure }\end{array}$ & \\
\hline Thickness/increment of axial slices & $2 / 1 \mathrm{~mm}$ & $3 / 1.5 \mathrm{~mm}$ \\
\hline MPRs & $\begin{array}{l}\text { requested in sagittal and coronal direction and paralle } \\
\text { to the long axis of the prox. extremities }\end{array}$ & \\
\hline
\end{tabular}

Abbreviations: No.: number; prox.: proximal; incl.: inclusive; PRM: persons with reduced mobility; kV: kilovoltage; mAs: milliampere-seconds; mm: millimeters; MPRs: multiplanar reconstructions

* With the limitation that attenuation measurements of peripheral intramedullary lesions should not be performed on bone kernel-reconstructed images that were set on the soft-tissue window, but on soft-tissue kernel-reconstructed images

infiltration before destruction of the mineralized bone tissue occurs [21]. MRI is considered to be the most sensitive and most specific imaging modality for noninvasive detection of early plasma cell invasion of the bone marrow, because it is able to evaluate medullary composition and cellularity [48]. Specific MRI findings may therefore prompt the upgrading of patients from precursor stages to active MM, even though the corresponding WBLDCT does not yet depict osteolysis (Fig. 3) [11, 49]. In addition, diffusion-weighted imaging (DWI) in WBMRI offers a functional imaging modality for the evaluation of the medullary bone, providing excellent accuracy for the differentiation of pathological from normal bone marrow. A meta-analysis from 2018 reported a pooled sensitivity of $89 \%$ and a pooled specificity of $87 \%$ of the $\mathrm{ADC}$, respectively, for the differentiation of benign and malignant vertebral bone marrow lesions [50].

In 2014, MRI findings were included in the diagnostic criteria of the IMWG. Since 2015, the IMWG recommends MRI as the gold standard for the imaging of the axial skeleton, the assessment of dolorous lesions and the differentiation of benign from malignant osteoporotic vertebral fractures [48]. Additionally, it is considered the method of choice to for the assessment of painful complications and spinal cord compression. Since 2016, the IMWG guidelines consider the detection of one or more focal lesions at least $5 \mathrm{~mm}$ in size on MRI as a criterion for active MM. Similar to the recommendations for WBLDCT, the finding of equivocal or smaller lesions on MRI should not be over-interpreted but should prompt a follow-up examination within 3-6 months in order to verify or exclude active MM. In contrast to the evidence of focal lesions as mentioned above, a diffuse infiltration pattern of the bone marrow on MRI is not yet considered a myeloma-defining event. According to the IMWG guidelines, it should prompt surveillance by follow-up imaging within 36 months, or MM may be confirmed with biopsy and histological evidence of $\geq 60 \%$ of plasma cells in the bone marrow specimen [25, 51].

Systematic WBMRI or alternatively MRI of the spine and the pelvis are further recommended by the IMWG as part of the initial assessment of asymptomatic patients and patients suffering from SMM [48, 52]. Focal lesions and diffuse medullary abnormalities are considered as suspect imaging features in these cases and are associated with a higher risk of progression to active MM [6]. They can be identified by MRI during the initial stage of disease, when osteolytic destruction is not yet evident on WBLDCT. Focal cortical or trabecular lesions at least $5 \mathrm{~mm}$ in diameter on MRI should be considered as criterion for symptomatic disease and initiation of therapy. A follow-up MRI after 3-6 months is further recommended for patients with SMM exhibiting equivocal focal lesions, as growth dynamics have additional predictive value, and SMM patients with progression, indicated by an increase in number or size of focal lesions, should be considered as 
Fig. 3 (a) Marked osteolytic lesions are detected in the 6th, 7th and 11th thoracic vertebral body and pathological fractures in the 7 th and 11 th vertebrae in the sagittal CT reconstruction of the spine in the bone window.

Additional lesions are suspected in the 8th-10th vertebral body. MRI of the patient using a T1weighted (b) and a STIR (c) sequence in sagittal orientation confirms the involvement of myeloma in the 6th, 7th and 11th vertebrae, while end plateassociated edema due to degenerative disease is seen in the 8th and 9th thoracic vertebrae. Thus, CT can sometimes overdiagnose myeloma involvement in cases of osteoporosis and inhomogeneous bone structure

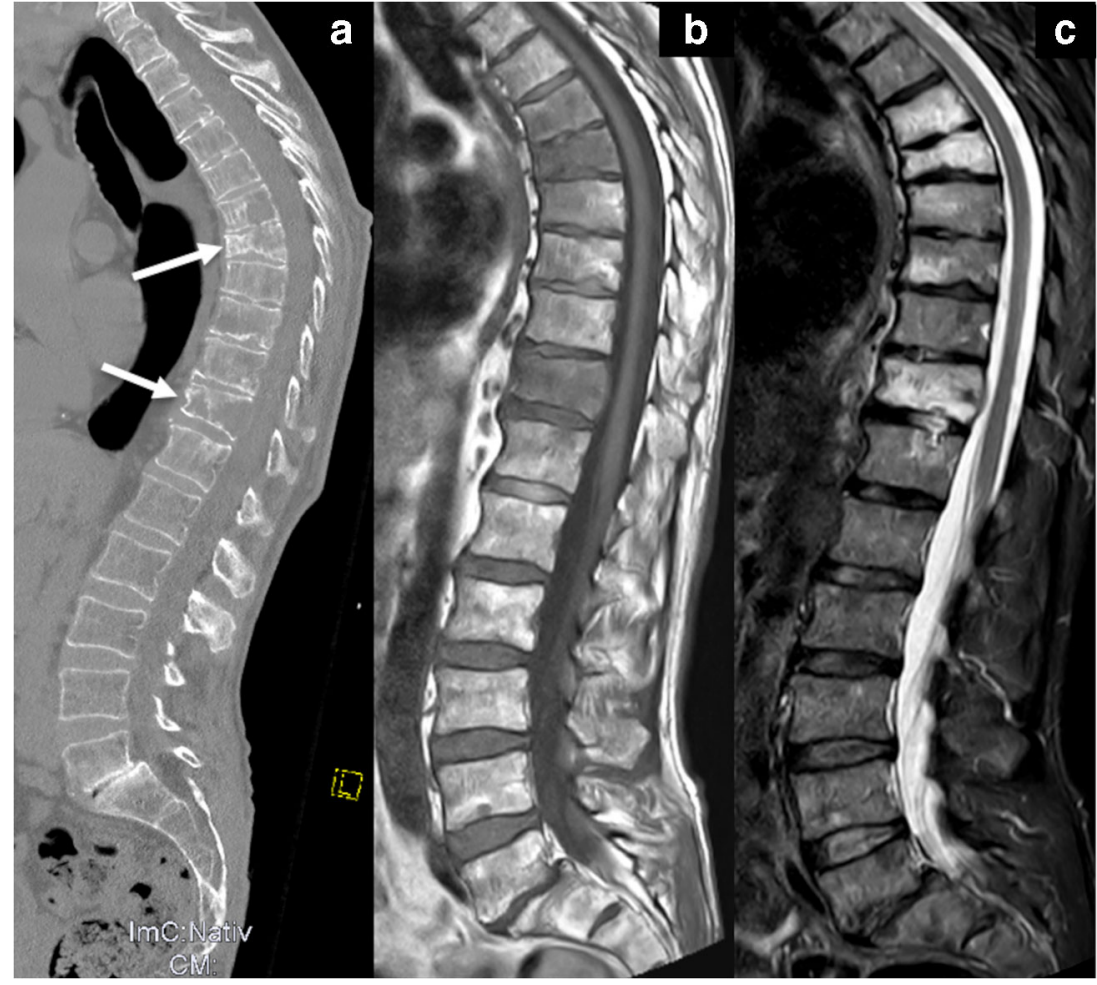

symptomatic and requiring therapy $[6,48]$. If $\mathrm{MRI}$ is unavailable, $\left[{ }^{18} \mathrm{~F}\right]-\mathrm{FDG}$ PET/CT is the method of choice to distinguish between SMM and active MM [18].

Systematic WBMRI is also proposed for the workup of patients with solitary bone plasmacytoma, while $\left[{ }^{18} \mathrm{~F}\right]-\mathrm{FDG}$ $\mathrm{PET} / \mathrm{CT}$ is recommended in cases of suspected solitary extramedullary plasmacytoma $[5,53]$. Solitary bone plasmacytoma is twice as prevalent and associated with a higher risk of progression to MM within the first 3 years [54]. The most important purpose of a WBMRI examination in solitary extramedullary plasmacytoma is the exclusion of additional bone marrow lesions and/or further soft-tissue masses, which differentiates this disease from systemic MM [25]. A second aspect is the detectability of a diffuse bone marrow infiltration, which may prompt further examinations and consequently lead to the upstaging of patients and modified treatment strategies [51]. Yearly follow-up examinations using the same imaging technique are recommended for the first 5 years after the initial diagnosis of solitary bone plasmacytoma.

In contrast, WBLDCT is recommended as first-line imaging modality in suspected high-risk non-IgM MGUS to rule out MM [25]. WBMRI is an accepted alternative if WBLDCT is not available and is helpful in the assessment of benign vertebral lesions, bone marrow edema and fractures in elderly patients. Furthermore, WBMRI or alternatively MRI of the spine and the pelvis are suggested in patients with equivocal findings on WBLDCT and suspected development of MM but no other apparent clinical evidence of myeloma such as hypercalcemia, renal failure, anemia and bone lesions, so called CRAB criteria [5].

\section{Imaging findings in WBMRI}

Imaging of bone marrow lesions in MM patients is inhomogeneous and differentiates between five patterns of plasma cell infiltration. They include (1) a normal appearance of the bone marrow despite minor microscopic plasma cell clusters, (2) focal lesions, which are defined as lesions of at least $5 \mathrm{~mm}$ in diameter, (3) a homogeneous diffuse infiltration, (4) a mixture of focal lesions and diffuse infiltration, and (5) a variegated or "salt-and-pepper" pattern due to an inhomogeneous bone marrow appearance with interposition of fat islands [10, $48,55,56]$. A high tumor burden is typically associated with a diffuse pattern or with focal lesions, while a normal or variegated MRI pattern is seen in patients with a low tumor burden [57]. Nevertheless, the IMWG has not yet defined the diffuse pattern as a criterion for symptomatic disease [48].

T1w SE sequences are the method of choice for the morphological evaluation of bone marrow, as suspicious, hypointense lesions contrast with the medullary fat that increases with increasing age. The lesions are usually as low as muscle signal intensity, whereas marrow edema is usually less hypointense. Sequences with fat suppression confirm suspicious lesions, which show a high signal intensity due to their high cellularity and water content. T2-weighted (T2w) SE sequences optimally visualize the spinal canal and enable the detection of bone and soft-tissue edema. In contrast to spectral 
fat saturation techniques, frequency-selective fast saturation techniques, such as short-tau inversion recovery (STIR), produce more homogeneous fat saturation in large fields of view. This is important for a whole-body setting (Fig. 4).

On DWI, signal intensity is high, with a low ADC, due to restriction of water protons in tumor nodules. Focal lesions enhance after the intravenous application of gadolinium. Suspicious focal bone lesions are easier to detect in elderly patients because of their higher medullary content of fat. It is important to realize that a focal bone marrow lesion on MRI is not automatically equal to an osteolytic lesion with bone destruction on CT. Focal medullary lesions on MRI simply illustrate a dense plasma cell infiltration that may be associated with a lytic lesion or that may precede bone destruction and the development of an osteolytic lesion. Thus, both imaging modalities, CT and MRI, provide complementary information and should be applied accordingly [5]. Corresponding guidelines for the implementation, acquisition and interpretation of WBMRI and WBLDCT have been developed [17, 25, 51].

Conversely, the detection of diffuse marrow infiltration sometimes remains challenging (Fig. 5). A diffuse medullary infiltration is characterized by a decreased signal on T1w sequences and a correspondingly increased signal on fatsaturated sequences. Low-grade diffuse disease $(<20$ vol\%) usually cannot be detected with MRI. In these initial stages, the fat component is often increased. Later, adipocytes are replaced by plasma cells, leading to a reduction in signal intensity on T1w SE images. T1w images have proven to be the most sensitive sequence for the evaluation of diffuse disease
[29]. In intermediate-grade diffuse bone marrow infiltration, the signal is reduced on T1w turbo spin-echo (TSE) images and eventually slightly increased on fat-saturated sequences. In high-grade plasmacytosis (> $50 \mathrm{vol} \%$ ), the signal is markedly decreased on T1w TSE images. The signal intensity of the marrow is almost the same as the intervertebral disc or as low as muscle. This was also described as the "bright disc" sign (Fig. 6) [29, 58, 59].

Contrast application can help in distinguishing normal from abnormal marrow, as contrast enhancement is higher in myeloma due to the high neovascularisation, at least in intermediate- and high-grade plasmacytosis [60, 61]. Red marrow reconversion has to be mentioned as a differential diagnosis in diffuse hypercellularity. It has been described early after initiation of chemotherapy, after administration of bone marrow-stimulating factors like erythropoietin or granulocyte colony-stimulating factor [14]. A reduced signal intensity in all MRI sequences due to medullary iron overload is another phenomenon that is frequently seen after stem cell transplantation or repeated blood transfusions, and which should be considered in patients suffering from an advanced stage of MM. However, here, the signal on T2w or fatsaturated sequences is also low [51].

In contrast to WBLDCT, MRI is primarily applied for neuroimaging in extraosseous MM [24, 62, 63]. MRI is considered the modality of choice when extraosseous soft-tissue masses are located in paraspinal or paravertebral areas, and it is the first method of choice whenever spinal cord compression is suspected $[48,64]$. Because of the brain-blood barrier,
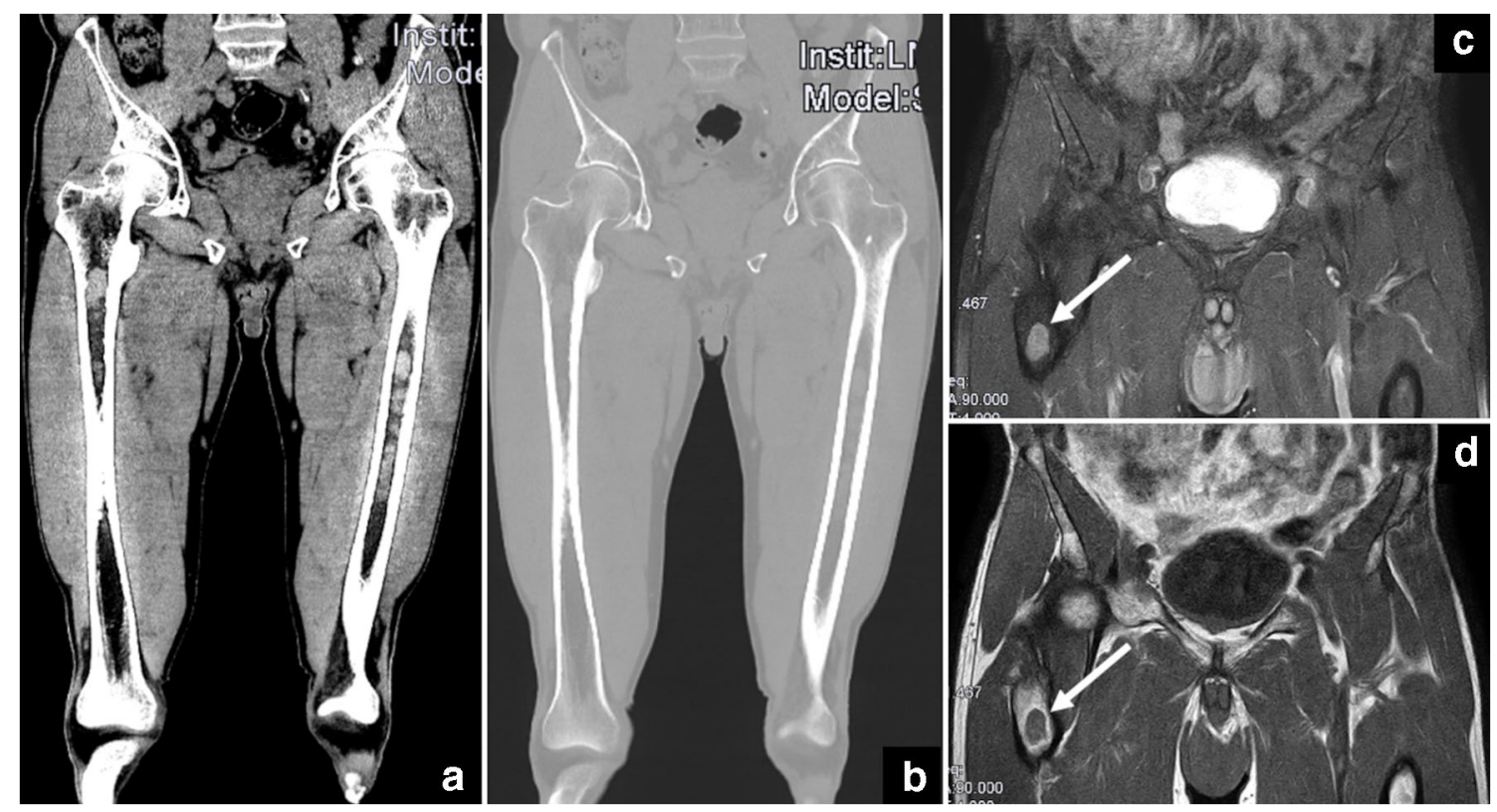

Fig. 4 Illustration of typical marrow infiltrates in both femurs. (a) Coronal CT reformation in the soft-tissue window setting shows focal lesions with high signal density $(75 \mathrm{HU})$ within the normal fatty marrow (black arrows). (b) The lesions can be missed in the corresponding CT reformation in the bone window setting. (c, d) The focal lesions exhibit a hypointense signal in the corresponding coronal T1w MRI scan and a hyperintense signal in the T1w fat-saturated scan (white arrows) 
Fig. 5 CT often underestimates tumor mass because on CT only myeloma infiltrates which have already led to osteolytic lesions can be detected. (a) The white arrows mark two osteolytic lesions in the 11th and 12th vertebral body on the sagittal CT reformation. The corresponding sagittal T1w MRI scan (b) and the STIR sequence (c) illustrate that every vertebral body is affected by myeloma
Fig. 6 MRI illustration of a diffuse medullary infiltration pattern in myeloma using T1w (a) and STIR (b) sequences. It is characterized by decreased signal intensity of the bone marrow on T1w sequences (a) and a correspondingly increased signal on STIR (b) sequences. On T1w images (a), the signal intensity of the bone marrow is equal to the intervertebral disc and the muscle, which is known as the "bright disc" sign
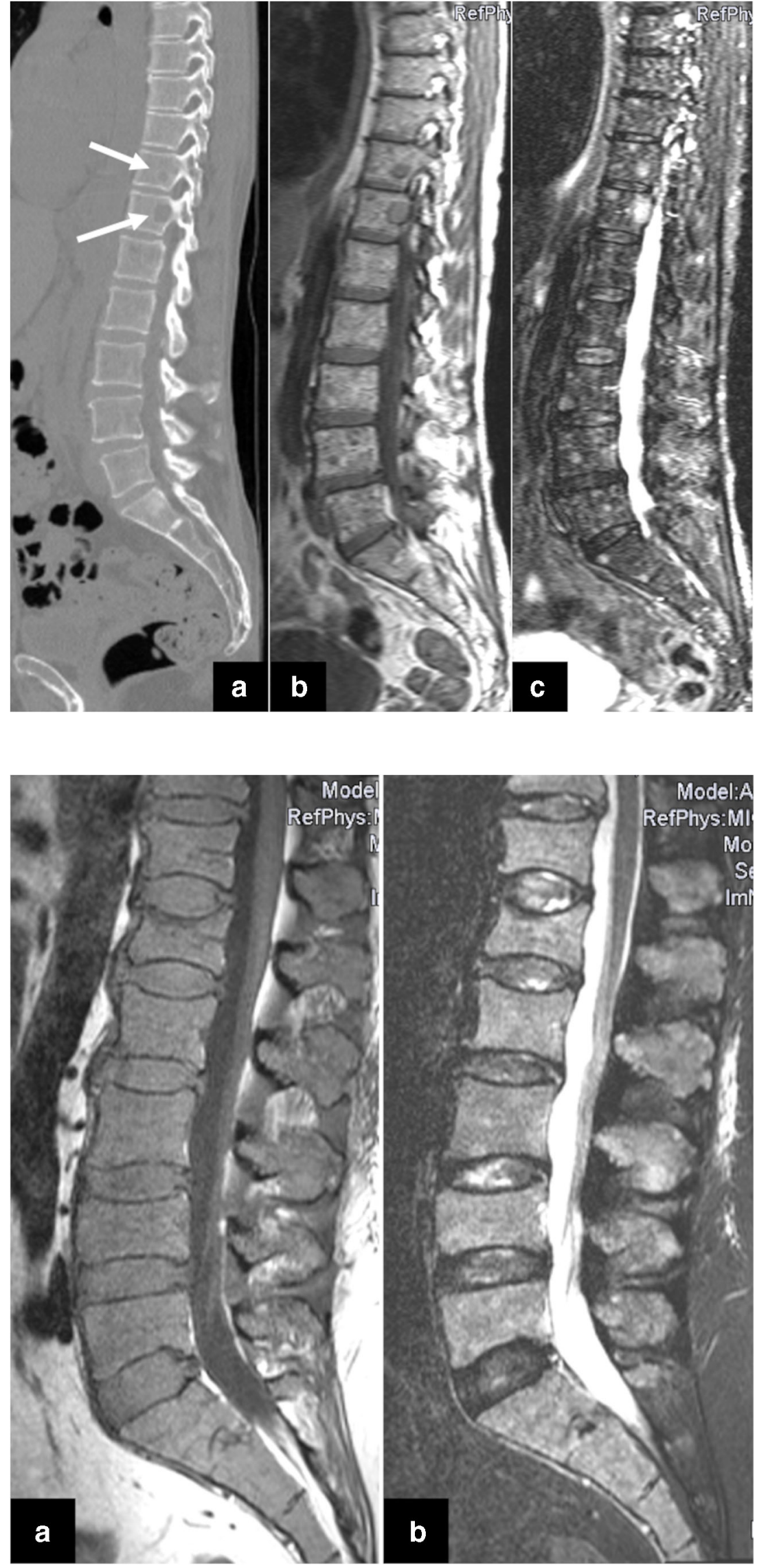
direct involvement of the central nervous system (CNS) is very rare, with an incidence of about $1 \%$ in MM patients. It usually develops during disease relapse or progression, either secondarily due to an expanding bone lesion or primarily by hematogenous spread or via meningeal myelomatosis $[63,65,66]$. Unenhanced and contrast-enhanced MRI is recommended in cases with neurological symptoms suggesting CNS manifestations, but image findings are nonspecific, and important differential diagnoses are drug toxicity and metabolic alterations [65].

The liver, spleen and lymph nodes are considered the most frequently affected abdominal sites in extraosseous MM. Due to the high soft-tissue contrast, MRI outperforms $\mathrm{CT}$ in the detection of a diffuse plasma cell infiltration of the parenchymatous abdominal organs, which is typically associated with hepato- or splenomegaly and is reported to be the most common pattern of manifestation on autopsy studies [32].

Recently, the incidence of CNS and extraosseous manifestations in patients suffering from MM seem to be increasing, perhaps due to modern therapies and more sensitive imaging techniques [67]. Both manifestation patterns are associated with poorer survival rates and prognosis, while the response to conventional treatment strategies remains poor [63, 67].

Apart from that, MRI is also able to reliably diagnose an avascular epiphyseal necrosis, which is a frequent incidental finding and complication of myeloma treatment. Functional and morphological MR imaging of the bone marrow microstructure and composition thereby enables the detection of preclinical ischemic stages identifying patients at risk prior to manifest bone infarcts [68]. This may reduce patients' distress and therapy-associated costs $[68,69]$.

\section{Advances in functional WBMRI}

\section{Diffusion-weighted MRI (DWI-MRI)}

Diffusion-weighted imaging (DWI) in WBMRI adds functional information to the morphological sequences. It is the most sensitive MRI sequence for the detection of metastatic bone lesions $[53,70]$ and it is quick to perform and interpret, and enables objective measurements [51,53]. As the ADC value is directly related to the tissue cell density, the signal intensity of the bone marrow on DWI and ADC maps during the course of the disease is associated with the amount of medullary plasma cell infiltration $[64,71]$.

DWI-MRI is superior to conventional sequences in the detection of spinal medullary lesions and is reliably able to differentiate between MM, SMM and MGUS [72]. Nevertheless, DWI-MRI lacks specificity when its findings are interpreted in isolation [51]. The technique is addressed in detail in a separate article in this special issue.

\section{Dynamic contrast-enhanced MRI (DCE-MRI)}

Dynamic contrast-enhanced MR imaging (DCE-MRI) is another advanced, functional technique that can be used for noninvasive evaluation of tumoral neoangiogenesis, perfusion characteristics, and tissue composition and permeability. It is based on a computer-assisted analysis of the distribution of gadolinium within the intra- and extracellular matrix in sequential images. Using ROIs, the changing patterns that occur during the passage of the contrast bolus are transformed into semiquantitative parameters that are plotted and expressed as time-intensity curves, which correlate with histological findings in MM. Additionally, a quantitative analysis can be performed using a pharmacokinetic mathematical model [71, 73-75]. At present, DCE-MRI is not recommended for the evaluation of bone marrow disease, but it may provide useful information about treated lesions and may be useful in the context of clinical trials investigating novel agents.

Five different types of time-intensity curves have been described in the literature. Medullary infiltration in MM is typically characterized by type 4 curves, which display a quick and steep wash-in with a rapid wash-out. Type 3 curves, which depict a quick and steep wash-in with a wash-out plateau, and type 5 curves, which represent a quick, but less intense wash-in and late stable or increasing enhancement, are less frequently seen in MM. Type 1 and 2 curves, which illustrate either no or a slow enhancement pattern, are characteristic for normal yellow bone marrow or are typically observed in patients with MGUS [74]. One study by Delorme et al. was able to show that even in low-grade diffuse disease and SMM, perfusion is increased in time-resolved contrastenhanced images when compared to healthy controls [76, 77].

DCE-MR images are acquired during and immediately after the intravenous administration of a bolus of contrast medium $(0.2 \mathrm{ml} / \mathrm{kg})$ at an injection rate of $5 \mathrm{ml} / \mathrm{s}$. Fast or ultrafast MRI sequences, such as gradient-echo (GRE) sequences with very short repetition and echo times, are recommended for the evaluation of early-contrast-enhancement kinetics, because they combine rapid sequential acquisition of 1-3 s per image with a reasonable temporal and spatial resolution. The fat-suppressed T1w ultrafast GRE sequence of the thoracolumbar spine is usually followed by static fat-suppressed $\mathrm{T} 1 \mathrm{w}$ images $[64,71]$.

\section{Chemical-shift MRI}

Chemical-shift-based fat-water separation imaging using $\mathrm{T} 1 \mathrm{w}$ GRE sequences is able to detect visceral and medullary fat deposits on in-phase images and may be used to calculate fat-fractioning images [78]. Therefore, the technique enables the detection of microscopic fat deposits within the bone marrow and the visceral organs because medullary fat and reservoirs of adipocytes, e.g., within the liver, exhibit a high signal intensity on in-phase images and a low signal on opposed- 
phase images. Moreover, chemical-shift imaging can be used to calculate fat-fraction images and opposed-phase images can aid the characterization of bone lesions [51]. In contrast to hemangiomas, which contain fat and thus are hyperintense on fat-selective images but hypointense on water-selective images, MM manifestations exhibit the opposite signal characteristics due to their high cellularity and water content.

Functional chemical-shift MRI sequences are useful in younger patients with medullary plasma cell infiltration but simultaneously a high content of hematopoietic bone marrow, and in cases with red marrow reconversion, during therapy because pathological conditions exhibit a signal decrease of more than $20 \%$ at opposed-phase examinations in comparison to in-phase MRI. In addition, functional chemical-shift MRI sequences are able to indicate medullary plasma cell infiltrates as low as $10 \%$ during the initial stage of disease, while conventional MRI sequences depict a normal bone marrow pattern $[21,79]$.

\section{Positioning and technical parameters of WBMRI}

As described above, there are several options for morphological and functional WB-MRI. In 2019, a multidisciplinary international expert group published current guidelines for the acquisition, interpretation and reporting of WBMRI in MM in order to standardize the diagnosis and to enable accurate assessment of the treatment response using the Myeloma Response Assessment and Diagnosis System (MY-RADS) [51].

The consensus for the technical prerequisites is that a field strength of $1.5 \mathrm{~T}$ should be used for WBMRI as a standard for imaging of MM due to its robustness and widespread availability [51]. Whenever possible, follow-up examinations should be performed on a similar unit. No specific patient preparation and no oral contrast administration is required for the acquisition of bone images. The patient lies supine on the MRI table in a headfirst position, and the head should rest in an appropriate head coil, while both arms should be positioned by the sides. Surface coils are recommended to maximize the signal-to-noise ratio of images, and they should cover the patient from the skull vertex to the mid-thigh without any gaps in between, to enable a precise evaluation. Wide anatomical coverage including the rib cage, the shoulder articulations and the long bones of the extremities is essential, as up to $50 \%$ of lesions may be missed by imaging the spine alone [80]. As WBMRI requires rather long scan times, attention should be paid to patient comfort and the most relevant sequences should be acquired first, since the risk of motion artifacts rises with increasing scan time. A general quality control program for MRI and periodic validation of ADC and fat fraction measurements is mandatory.

Studies should always include a combination of two morphological sequences (T1w and STIR) and at least one functional imaging sequence. The most important functional imaging sequence of choice is DWI, which should be included in every study, while DCE-MRI and chemical-shift MRI sequences are secondary to that. The recommended core clinical protocol should take about $30 \mathrm{~min}$, and is designed for the detection of the disease. The comprehensive protocol is required for the examination of soft tissue, extramedullary disease and patients with periodic tumor response assessments. It takes about 45-50 min. Detailed information about the imaging protocol is provided in Table 2, and a summary of the

Table 2 Summary of the recommended technical parameters and accepted alternatives for the WBMRI examination in MM [51]

\begin{tabular}{|c|c|c|c|c|c|}
\hline Sequence & Orientation & FOV & Section thickness/ technical details & Aim/ target structure & Protocol \\
\hline 1) T1w SE or TSE & Sagittal & wh.-sp. & $4-5 \mathrm{~mm}$ & Bone marrow & $*, \#$ \\
\hline 2) STIR or T2w SE fs or T2w & Sagittal & wh.-sp. & $4-5 \mathrm{~mm}$ & $\begin{array}{l}\text { Spinal canal, } \\
\text { bone/soft-tissue edema }\end{array}$ & $*, \#$ \\
\hline 3) T1w gradient-echo or T1w TSE & Sagittal/axial & wh.-bd. & $5 \mathrm{~mm}$ or thin, isotropic & Fat fraction maps & $*, \#$ \\
\hline $\begin{array}{l}\text { 4) DWI SE-EPI, ADC calc., 3D MIP } \\
\text { recon. of highest b-value images }\end{array}$ & Axial & wh.-bd. & $\begin{array}{l}5 \text { mm contiguous sectioning/2 } \\
\text { b-values (b50-100, } \\
\left.\text { b800-1000 s/mm } \mathrm{mm}^{2}\right)\end{array}$ & Bone marrow & $\begin{array}{l}\text { *, \# (additional } \\
\text { b500-600 s/- } \\
\mathrm{mm}^{2} \text { ) }\end{array}$ \\
\hline 5) T2w TSE & Axial & wh.-bd. & $\begin{array}{l}5 \mathrm{~mm} \text { contiguous sectioning/multiple } \\
\text { stations, preferably matching DWI }\end{array}$ & & *optional, \# \\
\hline 6) Local assessment & Individual & & & $\begin{array}{l}\text { Symptomatic/known sites } \\
\text { outside standard FOV }\end{array}$ & \#optional \\
\hline \multirow{2}{*}{\multicolumn{6}{|c|}{$\begin{array}{l}\text { Abbreviations: FOV: field of view; T1w: T1-weighted; SE: spin-echo; TSE: turbo spin-echo; wh.-sp.: whole spine; mm: millimeters; T2w: T2-weighted } \\
\text { GRE: gradient-echo; wh.-bd.: whole-body = vertex to knees; DWI: diffusion-weighted imaging; EPI: echo-planar imaging; calc.: calculation; ADC } \\
\text { apparent diffusion coefficient; MIP: maximum intensity projection; recon.: reconstruction; s: seconds; 3D: 3-dimensional; STIR: short tau inversion } \\
\text { recovery; fs: fat-saturated } \\
{ }^{\circ} \text { STIR or T2w FS preferred over T2w TSE, due to higher signal contrast }\end{array}$}} \\
\hline & & & & & \\
\hline \multicolumn{6}{|c|}{ * Recommended as part of the core clinical protocol } \\
\hline Recommended as part of the comp & ensive prot & & & & \\
\hline
\end{tabular}


imaging recommendations of the IMWG is provided in Tables 3 and 4.

\section{Limitations of WBMRI}

Restrictions that have to be addressed include the higher cost but lower availability of WBMRI when compared with WBLDCT. The more time-consuming WB examination may be challenging for patients with active MM and MBD who suffer from bone pain, as they are asked to lie as motionless as possible during image acquisition. Otherwise, motion artifacts may impede the interpretation of MR images. Claustrophobia is an additional serious limitation, which may be worsened by the application of body matrix anterior coils or the psychological aftereffects of the serious cancer diagnosis and rather intensive therapeutic treatments such as chemotherapy or stem cell transplantation. Although the number MRI-compatible metallic implants and medical devices is increasing, the technique is still contraindicated in patients with several older or incompatible models, due to the risk of device failure and heating.

Additionally, renal impairment is a frequent complication of myeloma, and restricted renal function is typically associated with a higher risk of complications in patients receiving MRI-contrast agents. A prospective trial by Hillengass et al. revealed no prognostic impact of the administration of gadopentetate-dimeglumine on progression-free survival in patients suffering from PCDs, when the current guidelines for the application of MRI contrast agents were respected [81]. Nevertheless, a significant adverse impact on kidney function was seen in symptomatic myeloma patients, who already exhibited reduced renal parameters at baseline, while patients with normal or slight renal impairment recovered.

In this context, the currently investigated and frequently discussed phenomenon of gadolinium deposition after serial

Table 3 Summary of the imaging recommendations of the IMWG [25] to establish the initial diagnosis and for the follow-up assessment of PCDs

\begin{tabular}{|c|c|c|}
\hline & WBLDCT & WBMRI or MRI of the spine and pelvis \\
\hline \multicolumn{3}{|c|}{ Imaging recommendations to establish the initial diagnosis } \\
\hline MGUS & To exclude MM in suspected high-risk non-IgM MGUS ${ }^{1}$ & $\begin{array}{l}\text { - Alternative, if CT is unavailable } \\
\text { - Next step to evaluate equivocal findings of CT }\end{array}$ \\
\hline SMM & Method of choice to detect osteolytic lesions** & $\begin{array}{l}\text { To exclude focal lesions as myeloma-defining } \\
\text { events, if CT is negative }\end{array}$ \\
\hline Solitary plasmacytoma & - & In solitary bone plasmacytoma ${ }^{3}$ \\
\hline MM & $\begin{array}{l}\text { Method of choice to detect and evaluate the } \\
\text { extend of osteolytic lesions }{ }^{4}\end{array}$ & $\begin{array}{l}\text { Next step to exclude focal lesions as myeloma-defining } \\
\text { events, if CT is negative and no other } \\
\text { myeloma-defining event is present }{ }^{5}\end{array}$ \\
\hline \multicolumn{3}{|c|}{ Imaging recommendations for follow-up examinations and the assessment of treatment response } \\
\hline MGUS & \multicolumn{2}{|c|}{ Not indicated unless signs of progression to symptomatic disease occur } \\
\hline SMM & \multicolumn{2}{|c|}{$\begin{array}{l}\text { - Repetition of same technique used at initial diagnosis at yearly intervals for at least } 5 \text { years depending on the risk factors } \\
\text { - Additional alternating WBLDCT in cases with a high risk of progression to identify small osteolytic lesions }\end{array}$} \\
\hline Solitary plasmacytoma & \multicolumn{2}{|c|}{ Repetition of same technique used at initial diagnosis at yearly intervals for at least 5 years } \\
\hline MM & \multicolumn{2}{|c|}{$\begin{array}{l}\text { - Repetition of same technique used at initial diagnosis to provide comparability } \\
\text { - Follow-up imaging should be adapted in cases of progression, when the repeated and initially applied imaging technique } \\
\text { does not reveal post-treatment imaging results that justify a change of treatment } \\
\text { - WBLDCT when a relapse is suspected to evaluate the extent and dynamics of bone destruction as the most clinically relevant } \\
\text { parameter }\end{array}$} \\
\hline
\end{tabular}

Abbreviations: WBLDCT: whole-body low-dose computed tomography; WBMRI: whole-body magnetic resonance imaging; MGUS: monoclonal gammopathy of undetermined significance is a plasma cell dyscrasia in which plasma cells or other types of antibody-producing cells secrete a myeloma protein, i.e., an abnormal antibody, into the blood; this abnormal protein is usually found during standard laboratory blood or urine tests; SMM: smoldering multiple myeloma (also sometimes known as asymptomatic myeloma) is an early form of myeloma, which usually progresses to active myeloma, but at a slow rate. In smoldering myeloma, abnormal cells can be detected in the bone marrow, and abnormal protein can be detected in the blood and/or urine; MM: multiple myeloma

${ }^{1}$ Additional PET/CT if WBLDCT is positive

2 PET/CT can be used as an alternative to WBLDCT and instead of WBMRI if the latter is unavailable or in cases with specific contraindications

${ }^{3} \mathrm{PET} / \mathrm{CT}$ in patients with solitary extramedullary plasmacytoma and as an alternative in solitary bone plasmacytoma, if MRI is unavailable

${ }^{4} \mathrm{PET} / \mathrm{CT}$ can be used as an alternative to WBLDCT

${ }^{5} \mathrm{PET} / \mathrm{CT}$ can be used as an alternative to MRI if the latter is unavailable or in cases with specific contraindications, and it is the preferred imaging method to generate a baseline for follow-up assessments

${ }^{6}$ Yearly follow-ups are recommended for patients with residual lesions detected by PET/CT because of the high risk of early progression 
Table 4 Summary and comparison of the main features, advantages and limitations of CT and MRI in MM and other PCDs

\begin{tabular}{|c|c|c|}
\hline & CT & MRI \\
\hline Main features & $\begin{array}{l}\text { - Morphological imaging in clinical routine, functional } \\
\text { imaging (DECT) only in study settings } \\
\text { - Highest sensitivity for the detection of osteolytic } \\
\text { bone lesions } \\
\text { - Visualization of bone marrow infiltration in the extremities } \\
\text { - Gold standard for the assessment of spinal stability } \\
\text { in vertebral fractures } \\
\text { - Necessary/supportive for the planning of radiotherapy, } \\
\text { surgeries and biopsies } \\
\text { - Valuable for the assessment of treatment response } \\
\text { and relapse during follow-up to evaluate the extent } \\
\text { and dynamics of bone destruction as the most } \\
\text { clinically relevant parameter }\end{array}$ & $\begin{array}{l}\text { - Morphological and functional imaging (DWI) } \\
\text { in clinical routine } \\
\text { - Additional functional imaging options (DCE, } \\
\text { chemical shift with fat fraction maps) in study settings } \\
\text { - Highest sensitivity for the detection of bone } \\
\text { marrow infiltration } \\
\text { - Depiction of medullary infiltration pattern } \\
\text { - Depiction of non-osteolytic bone marrow infiltration } \\
\text { prior to bone destruction } \\
\text { - Gold standard for the assessment of spinal cord } \\
\text { and nerve root compression } \\
\text { - Gold standard for the differentiation of benign } \\
\text { and malignant vertebral fractures } \\
\text { - Prognostic relevance } \\
\text { - Possible value for the assessment of treatment } \\
\text { response and relapse during follow-up when } \\
\text { functional imaging (DWI) is applied }\end{array}$ \\
\hline Technical advantages & $\begin{array}{l}\text { - Better availability } \\
\text { - Shorter acquisition time } \\
\text { - Higher patient comfort } \\
\text { - Lower costs } \\
\text { - No administration of contrast agents required } \\
\quad \text { for evaluation of MBD }\end{array}$ & No radiation exposure \\
\hline Technical limitations & - Radiation exposure & $\begin{array}{l}\text { - Limited availability } \\
\text { - Longer acquisition time } \\
\text { - Patient discomfort, may be significant in patients } \\
\quad \text { with claustrophobia, or older metal implants }\end{array}$ \\
\hline $\begin{array}{l}\text { Administration of } \\
\text { contrast agents }\end{array}$ & $\begin{array}{l}\text { - Adverse impact on kidney function in patients } \\
\text { with preexistent renal impairment } \\
\text { - Possible allergic reactions } \\
\text { - Possible thyrotoxicosis }\end{array}$ & $\begin{array}{l}\text { - Adverse impact on kidney function in patients } \\
\text { with preexistent renal impairment } \\
\text { - Disassociation and deposition of linear GBCAs }\end{array}$ \\
\hline
\end{tabular}

Abbreviations: CT: computed tomography; MRI: magnetic resonance imaging; DECT: dual-energy computed tomography; DWI: diffusion-weighted imaging; DCE: dynamic contrast-enhanced; GBCAs: gadolinium-based contrast agents

injections of linear gadolinium-based contrast agents (GBCAs) needs to be addressed. Clinical data regarding the deposition in extracranial tissues, especially in the bone and the bone marrow, are rare [82]. A recently published meta-analysis from preclinical and clinical pharmacokinetic data confirmed the effect of prolonged excretion of linear GBCAs and thereby indirectly demonstrated the existence of a deep compartment of distribution for gadolinium in the body [83]. This is especially true for linear GBCAs. However, no specific clinical symptoms possibly related to toxic effects of the accumulation have been observed so far, and no specific interference with the imaging findings in the bone during periodic staging MRI examinations have been reported thus far [82].

\section{Summary}

Imaging in the diagnosis, follow-up and evaluation of complications in MM has evolved and improved tremendously over the past two decades.
WBLDCT has replaced the WBXR survey and enables the highly sensitive detection of osteolytic lesions in MBD. It is the first method of choice for morphological imaging of patients suffering from myeloma in clinical routine, and the gold standard for the assessment of spinal stability in MBDassociated vertebral fractures. The planning of surgical device implantation and the guiding of biopsies in focal lesions are further indications for a dedicated CT exam. The IMWG now recommends a WBLDCT examination in high-risk patients suffering from non-IgM MGUS and in SMM in order to exclude osteolytic bone destruction as features of active MM.

WBMRI is the alternative to WBLDCT, if CT is unavailable, and is the method of choice to evaluate equivocal findings of CT. WBMRI reliably visualizes early medullary plasma cell invasion before bone destruction. In MM-associated complications and extraosseous manifestations, MRI is the first method of choice in patients with suspected spinal cord compression, and DWI-MRI is considered the most sensitive technique for the differentiation of benign osteoporotic and malignant vertebral compression fractures. WBMRI is further 
recommended in SMM patients with negative or inconclusive CT results to rule out two or more focal lesions, which are considered a myeloma-defining event. Functional MRI provides information on lesion composition in active MM with consequent prognostic implications and offers a high potential for the assessment of treatment response.

The recommendations for the acquisition, interpretation and reporting of WBLDCT and the corresponding MYRADS recommendations for MRI enable a standardized systemic application of both imaging modalities in clinical routine across various locations, thereby creating a fundamental cornerstone for multicenter trials.

\begin{abstract}
Abbreviations ADC, Apparent diffusion coefficient; CNS, Central nervous system; CT, Computed tomography; DCE MRI, Dynamic contrastenhanced magnetic resonance imaging; DECT, Dual-energy computed tomography; DWI-MRI, Diffusion-weighted imaging magnetic resonance imaging; $\left[{ }^{18} \mathrm{~F}\right]-\mathrm{FDG}$, [Fluorine-18]-Fluorodeoxyglucose; GBCA, Gadolinium-based contrast agents; GRE , Gradient-echo; IMWG, International Myeloma Working Group; MBD, Myeloma-associated bone disease; MGUS, Monoclonal gammopathy of undetermined significance; MM, Multiple myeloma; MRI, Magnetic resonance imaging; MY-RADS, Myeloma Response Assessment and Diagnosis System; PCD, Plasma cell disorders; PET/CT, Positron emission tomography computed tomography; SE, Spin-echo; STIR, Short-tau inversion recovery; SMM, Smoldering multiple myeloma; T1w, T1-weighted; T2w, T2weighted; TSE , Turbo spin-echo; VNCa images, Virtual non-calcium images; WBCT, Whole-body computed tomography; WBLDCT, Whole-body low dose computed tomography; WBMRI, Whole body magnetic resonance imaging; WBXR, Whole-body radiographic skeletal survey; XR, Conventional radiography
\end{abstract}

Funding Open Access funding enabled and organized by Projekt DEAL.

\section{Declarations}

Conflict of interest For all authors, no conflicts of interest were declared.

Open Access This article is licensed under a Creative Commons Attribution 4.0 International License, which permits use, sharing, adaptation, distribution and reproduction in any medium or format, as long as you give appropriate credit to the original author(s) and the source, provide a link to the Creative Commons licence, and indicate if changes were made. The images or other third party material in this article are included in the article's Creative Commons licence, unless indicated otherwise in a credit line to the material. If material is not included in the article's Creative Commons licence and your intended use is not permitted by statutory regulation or exceeds the permitted use, you will need to obtain permission directly from the copyright holder. To view a copy of this licence, visit http://creativecommons.org/licenses/by/4.0/.

\section{References}

1. Rajkumar SV. Updated diagnostic criteria and staging system for multiple myeloma. Am Soc Clin Oncol Educ Book. 2016;35:e41823.
2. Baffour FI, Glazebrook KN, Kumar SK, Broski SM. Role of imaging in multiple myeloma. Am J Hematol. 2020;95(8):966-77.

3. Kumar SK, Rajkumar SV. The multiple myelomas - current concepts in cytogenetic classification and therapy. Nat Rev Clin Oncol. 2018;15(7):409-21.

4. Kumar SK, Rajkumar V, Kyle RA, van Duin M, Sonneveld P, Mateos MV, et al. Multiple myeloma. Nat Rev Dis Primers. 2017;3:17046.

5. Rasch S, Lund T, Asmussen JT, Lerberg Nielsen A, Faebo Larsen R, Osterheden Andersen M, et al. Multiple Myeloma Associated Bone Disease. Cancers (Basel). 2020;12:8.

6. Rajkumar SV, Dimopoulos MA, Palumbo A, Blade J, Merlini G, Mateos MV, et al. International myeloma working group updated criteria for the diagnosis of multiple myeloma. Lancet Oncol. 2014;15(12):e538-48.

7. Melton LJ 3rd, Kyle RA, Achenbach SJ, Oberg AL, Rajkumar SV. Fracture risk with multiple myeloma: a population-based study. J Bone Miner Res. 2005;20(3):487-93.

8. Dimopoulos M, Terpos E, Comenzo RL, Tosi P, Beksac M, Sezer $\mathrm{O}$, et al. International myeloma working group consensus statement and guidelines regarding the current role of imaging techniques in the diagnosis and monitoring of multiple myeloma. Leukemia. 2009;23(9):1545-56.

9. Terpos E, Moulopoulos LA, Dimopoulos MA. Advances in imaging and the management of myeloma bone disease. J Clin Oncol. 2011;29(14):1907-15.

10. Baur-Melnyk A, Reiser M. Staging of multiple myeloma with MRI: comparison to MSCT and conventional radiography. Radiologe. 2004;44(9):874-81.

11. Baur-Melnyk A, Buhmann S, Becker C, Schoenberg SO, Lang N, Bartl R, et al. Whole-body MRI versus whole-body MDCT for staging of multiple myeloma. AJR Am J Roentgenol. 2008;190(4):1097-104.

12. Kumar S, Glazebrook KN, Broski SM. Fludeoxyglucose F 18 PET/ computed tomography evaluation of therapeutic response in multiple myeloma. PET Clin. 2019;14(3):391-403.

13. Kumar S, Paiva B, Anderson KC, Durie B, Landgren O, Moreau P, et al. International myeloma working group consensus criteria for response and minimal residual disease assessment in multiple myeloma. Lancet Oncol. 2016;17(8):e328-46.

14. Di Giuliano F, Picchi E, Muto M, Calcagni A, Ferrazzoli V, Da Ros $\mathrm{V}$, et al. Radiological imaging in multiple myeloma: review of the state-of-the-art. Neuroradiology. 2020;62(8):905-23.

15. Hillengass J, Moulopoulos LA, Delorme S, Koutoulidis V, Mosebach J, Hielscher T, et al. Whole-body computed tomography versus conventional skeletal survey in patients with multiple myeloma: a study of the international myeloma working group. Blood Cancer J. 2017;7(8):e599.

16. Moreau P, San Miguel J, Sonneveld P, Mateos MV, Zamagni E, Avet-Loiseau H, et al. Multiple myeloma: ESMO Clinical Practice Guidelines for diagnosis, treatment and follow-up. Ann Oncol. 2017;28(suppl_4):iv52-61.

17. Moulopoulos LA, Koutoulidis V, Hillengass J, Zamagni E, Aquerreta JD, Roche CL, et al. Recommendations for acquisition, interpretation and reporting of whole body low dose CT in patients with multiple myeloma and other plasma cell disorders: a report of the IMWG bone working group. Blood Cancer J. 2018;8(10):95.

18. Cavo M, Terpos E, Nanni C, Moreau P, Lentzsch S, Zweegman S, et al. Role of (18)F-FDG PET/CT in the diagnosis and management of multiple myeloma and other plasma cell disorders: a consensus statement by the international myeloma working group. Lancet Oncol. 2017;18(4):e206-17.

19. Rajkumar SV. Evolving diagnostic criteria for multiple myeloma. Hematol Am Soc Hematol Educ Program. 2015;2015:272-8. 
20. Amini B, Yellapragada S, Shah S, Rohren E, Vikram R. State-ofthe-art imaging and staging of plasma cell Dyscrasias. Radiol Clin N Am. 2016;54(3):581-96.

21. Ormond Filho AG, Carneiro BC, Pastore D, Silva IP, Yamashita SR, Consolo FD, et al. Whole-Body imaging of multiple myeloma: diagnostic criteria. Radiographics. 2019;39(4):1077-97.

22. Spira D, Weisel K, Brodoefel H, Schulze M, Kaufmann S, Horger M. Can whole-body low-dose multidetector CT exclude the presence of myeloma bone disease in patients with monoclonal gammopathy of undetermined significance (MGUS)? Acad Radiol. 2012;19(1):89-94.

23. Kyle RA, Larson DR, Therneau TM, Dispenzieri A, Kumar S, Cerhan JR, et al. Long-term follow-up of monoclonal Gammopathy of undetermined significance. N Engl J Med. 2018;378(3):241-9.

24. Lasocki A, Gaillard F, Harrison SJ. Multiple myeloma of the spine. Neuroradiol J. 2017;30(3):259-68.

25. Hillengass J, Usmani S, Rajkumar SV, Durie BGM, Mateos MV, Lonial S, et al. International myeloma working group consensus recommendations on imaging in monoclonal plasma cell disorders. Lancet Oncol. 2019;20(6):e302-12.

26. Horger M, Pereira P, Claussen CD, Kanz L, Vonthein R, Denecke $\mathrm{B}$, et al. Hyperattenuating bone marrow abnormalities in myeloma patients using whole-body non-enhanced low-dose MDCT: correlation with haematological parameters. Br J Radiol. 2008;81(965): 386-96.

27. Nishida Y, Matsue Y, Suehara Y, Fukumoto K, Fujisawa M, Takeuchi M, et al. Clinical and prognostic significance of bone marrow abnormalities in the appendicular skeleton detected by low-dose whole-body multidetector computed tomography in patients with multiple myeloma. Blood Cancer J. 2015;5:e329.

28. Zambello R, Crimi F, Lico A, Barila G, Branca A, Guolo A, et al. Whole-body low-dose CT recognizes two distinct patterns of lytic lesions in multiple myeloma patients with different disease metabolism at PET/MRI. Ann Hematol. 2019;98(3):679-89.

29. Baur A, Stabler A, Steinborn M, Schnarkowski P, Pistitsch C, Lamerz R, et al. Magnetic resonance tomography in plasmacytoma: ranking of various sequences in diffuse and focal infiltration patterns. Rofo. 1998;168(4):323-9.

30. Baur A, Stabler A, Bartl R, Lamerz R, Scheidler J, Reiser M. MRI gadolinium enhancement of bone marrow: age-related changes in normals and in diffuse neoplastic infiltration. Skelet Radiol. 1997;26(7):414-8

31. Varettoni M, Corso A, Pica G, Mangiacavalli S, Pascutto C, Lazzarino M. Incidence, presenting features and outcome of extramedullary disease in multiple myeloma: a longitudinal study on 1003 consecutive patients. Ann Oncol. 2010;21(2):325-30.

32. Cho R, Myers DT, Onwubiko IN, Williams TR. Extraosseous multiple myeloma: imaging spectrum in the abdomen and pelvis. Abdom Radiol (NY). 2020;46(3):1194-209.

33. Mosebach J, Thierjung H, Schlemmer HP, Delorme S. Multiple myeloma guidelines and their recent updates: implications for imaging. Rofo. 2019;191(11):998-1009.

34. Terpos E, Berenson J, Cook RJ, Lipton A, Coleman RE. Prognostic variables for survival and skeletal complications in patients with multiple myeloma osteolytic bone disease. Leukemia. 2010;24(5): 1043-9.

35. Lecouvet FE, Vande Berg BC, Malghem J, Maldague BE. Magnetic resonance and computed tomography imaging in multiple myeloma. Semin Musculoskelet Radiol. 2001;5(1):43-55.

36. Horger M, Claussen CD, Bross-Bach U, Vonthein R, Trabold T, Heuschmid M, et al. Whole-body low-dose multidetector row-CT in the diagnosis of multiple myeloma: an alternative to conventional radiography. Eur J Radiol. 2005;54(2):289-97.

37. Gleeson TG, Byrne B, Kenny P, Last J, Fitzpatrick P, O'Gorman P, et al. Image quality in low-dose multidetector computed tomography: a pilot study to assess feasibility and dose optimization in whole-body bone imaging. Can Assoc Radiol J. 2010;61(5): 258-64.

38. Gleeson TG, Moriarty J, Shortt CP, Gleeson JP, Fitzpatrick P, Byrne B, et al. Accuracy of whole-body low-dose multidetector CT (WBLDCT) versus skeletal survey in the detection of myelomatous lesions, and correlation of disease distribution with wholebody MRI (WBMRI). Skelet Radiol. 2009;38(3):225-36.

39. Lambert L, Ourednicek P, Meckova Z, Gavelli G, Straub J, Spicka I. Whole-body low-dose computed tomography in multiple myeloma staging: superior diagnostic performance in the detection of bone lesions, vertebral compression fractures, rib fractures and extraskeletal findings compared to radiography with similar radiation exposure. Oncol Lett. 2017;13(4):2490-4.

40. Suntharalingam S, Mikat C, Wetter A, Guberina N, Salem A, Heil $\mathrm{P}$, et al. Whole-body ultra-low dose CT using spectral shaping for detection of osteolytic lesion in multiple myeloma. Eur Radiol. 2018;28(6):2273-80.

41. Baldi D, Tramontano L, Alfano V, Punzo B, Cavaliere C, Salvatore M. Whole Body low dose computed tomography using thirdgeneration dual-source multidetector with spectral shaping: protocol optimization and literature review. Dose Response. 2020;18(4): 1559325820973131.

42. Shan H, Zhang Y, Yang Q, Kruger U, Kalra MK, Sun L, et al. 3-D convolutional encoder-decoder network for low-dose CT via transfer learning from a 2-D trained network. IEEE Trans Med Imaging. 2018;37(6):1522-34.

43. Thomsen FSL, Delrieux CA, Pisula JI, Fuertes Garcia JM, Lucena $\mathrm{M}$, de Luis GR, et al. Noise reduction using novel loss functions to compute tissue mineral density and trabecular bone volume fraction on low resolution QCT. Comput Med Imaging Graph. 2020;86: 101816.

44. Borggrefe J, Giravent S, Thomsen F, Pena J, Campbell G, Wulff A, et al. Association of QCT bone mineral density and bone structure with vertebral fractures in patients with multiple myeloma. J Bone Miner Res. 2015;30(7):1329-37.

45. Kosmala A, Weng AM, Krauss B, Knop S, Bley TA, Petritsch B. Dual-energy CT of the bone marrow in multiple myeloma: diagnostic accuracy for quantitative differentiation of infiltration patterns. Eur Radiol. 2018;28(12):5083-90.

46. Kosmala A, Weng AM, Heidemeier A, Krauss B, Knop S, Bley TA, et al. Multiple myeloma and dual-energy CT: diagnostic accuracy of virtual noncalcium technique for detection of bone marrow infiltration of the spine and pelvis. Radiology. 2018;286(1):205-13.

47. Thomas C, Schabel C, Krauss B, Weisel K, Bongers M, Claussen $\mathrm{CD}$, et al. Dual-energy CT: virtual calcium subtraction for assessment of bone marrow involvement of the spine in multiple myeloma. AJR Am J Roentgenol. 2015;204(3):W324-31.

48. Dimopoulos MA, Hillengass J, Usmani S, Zamagni E, Lentzsch S, Davies FE, et al. Role of magnetic resonance imaging in the management of patients with multiple myeloma: a consensus statement. J Clin Oncol. 2015;33(6):657-64.

49. Baur A, Stabler A, Nagel D, Lamerz R, Bartl R, Hiller E, et al. Magnetic resonance imaging as a supplement for the clinical staging system of Durie and Salmon? Cancer. 2002;95(6):1334-45.

50. Suh CH, Yun SJ, Jin W, Lee SH, Park SY, Ryu CW. ADC as a useful diagnostic tool for differentiating benign and malignant vertebral bone marrow lesions and compression fractures: a systematic review and meta-analysis. Eur Radiol. 2018;28(7):2890-902.

51. Messiou C, Hillengass J, Delorme S, Lecouvet FE, Moulopoulos LA, Collins DJ, et al. Guidelines for acquisition, interpretation, and reporting of Whole-Body MRI in myeloma: myeloma response assessment and diagnosis system (MY-RADS). Radiology. 2019;291(1):5-13.

52. Lecouvet FE, Van Nieuwenhove S, Jamar F, Lhommel R, Guermazi A, Pasoglou VP. Whole-Body MR imaging: the novel, 
"intrinsically hybrid," approach to metastases, myeloma, lymphoma, in bones and beyond. PET Clin. 2018;13(4):505-22.

53. Lecouvet FE, Whole-Body MR. Imaging: musculoskeletal applications. Radiology. 2016;279(2):345-65.

54. Nahi H, Genell A, Walinder G, Uttervall K, Juliusson G, Karin F, et al. Incidence, characteristics, and outcome of solitary plasmacytoma and plasma cell leukemia. Population-based data from the Swedish myeloma register. Eur J Haematol. 2017;99(3): 216-22.

55. Baur-Melnyk A, Buhmann S, Durr HR, Reiser M. Role of MRI for the diagnosis and prognosis of multiple myeloma. Eur J Radiol. 2005;55(1):56-63.

56. Baur A, Stabler A, Bartl R, Lamerz R, Reiser M. Infiltration patterns of plasmacytomas in magnetic resonance tomography. Rofo. 1996;164(6):457-63.

57. Stabler A, Baur A, Bartl R, Munker R, Lamerz R, Reiser MF. Contrast enhancement and quantitative signal analysis in MR imaging of multiple myeloma: assessment of focal and diffuse growth patterns in marrow correlated with biopsies and survival rates. AJR Am J Roentgenol. 1996;167(4):1029-36.

58. Delorme S, Baur-Melnyk A. Imaging in multiple myeloma. Eur J Radiol. 2009;70(3):401-8.

59. Schmidt GP, Baur A, Stabler A, Schoenberg SO, Steinborn M, Baltin V, et al. Estimation of diffuse bone marrow infiltration of the spine in multiple myeloma: correlation of MRT with histological results. Rofo. 2005;177(5):745-50.

60. Baur A, Bartl R, Pellengahr C, Baltin V, Reiser M. Neovascularization of bone marrow in patients with diffuse multiple myeloma: a correlative study of magnetic resonance imaging and histopathologic findings. Cancer. 2004;101(11):2599-604.

61. Nosas-Garcia S, Moehler T, Wasser K, Kiessling F, Bartl R, Zuna I, et al. Dynamic contrast-enhanced MRI for assessing the disease activity of multiple myeloma: a comparative study with histology and clinical markers. J Magn Reson Imaging. 2005;22(1):154-62.

62. Agarwal A. Neuroimaging of plasmacytoma. A pictorial review. Neuroradiol J. 2014;27(4):431-7.

63. Lasocki A, Gangatharan S, Gaillard F, Harrison SJ. Intracranial involvement by multiple myeloma. Clin Radiol. 2015;70(8):890-7.

64. Dutoit JC, Verstraete KL. Whole-body MRI, dynamic contrastenhanced MRI, and diffusion-weighted imaging for the staging of multiple myeloma. Skelet Radiol. 2017;46(6):733-50.

65. Cerase A, Tarantino A, Gozzetti A, Muccio CF, Gennari P, Monti $\mathrm{L}$, et al. Intracranial involvement in plasmacytomas and multiple myeloma: a pictorial essay. Neuroradiology. 2008;50(8):665-74.

66. Gozzetti A, Cerase A, Defina M, Bocchia M. Plasmacytoma of the skull. Eur J Haematol. 2012;88(4):369.

67. Gangatharan SA, Carney DA, Prince HM, Wolf MM, Januszewicz EH, Ritchie DS, et al. Emergence of central nervous system myeloma in the era of novel agents. Hematol Oncol. 2012;30(4):170-4.

68. Fu W, Liu B, Wang B, Zhao D. Early diagnosis and treatment of steroid-induced osteonecrosis of the femoral head. Int Orthop. 2019;43(5):1083-7.

69. Murphey MD, Foreman KL, Klassen-Fischer MK, Fox MG, Chung EM, Kransdorf MJ. From the radiologic pathology archives imaging of osteonecrosis: radiologic-pathologic correlation. Radiographics. 2014;34(4):1003-28.

70. Wu LM, Gu HY, Zheng J, Xu X, Lin LH, Deng X, et al. Diagnostic value of whole-body magnetic resonance imaging for bone metastases: a systematic review and meta-analysis. J Magn Reson Imaging. 2011;34(1):128-35.

71. Dutoit JC, Verstraete KL. MRI in multiple myeloma: a pictorial review of diagnostic and post-treatment findings. Insights Imaging. 2016;7(4):553-69.

72. Dutoit JC, Vanderkerken MA, Anthonissen J, Dochy F, Verstraete KL. The diagnostic value of SE MRI and DWI of the spine in patients with monoclonal gammopathy of undetermined significance, smouldering myeloma and multiple myeloma. Eur Radiol. 2014;24(11):2754-65.

73. Hillengass J, Landgren O. Challenges and opportunities of novel imaging techniques in monoclonal plasma cell disorders: imaging "early myeloma". Leuk Lymphoma. 2013;54(7):1355-63.

74. Dutoit JC, Vanderkerken MA, Verstraete KL. Value of whole body MRI and dynamic contrast enhanced MRI in the diagnosis, followup and evaluation of disease activity and extent in multiple myeloma. Eur J Radiol. 2013;82(9):1444-52.

75. Schmidt GP, Reiser MF, Baur-Melnyk A. Whole-body MRI for the staging and follow-up of patients with metastasis. Eur J Radiol. 2009;70(3):393-400.

76. Hillengass J, Ritsch J, Merz M, Wagner B, Kunz C, Hielscher T, et al. Increased microcirculation detected by dynamic contrastenhanced magnetic resonance imaging is of prognostic significance in asymptomatic myeloma. Br J Haematol. 2016;174(1):127-35.

77. Hillengass J, Zechmann C, Bauerle T, Wagner-Gund B, Heiss C, Benner A, et al. Dynamic contrast-enhanced magnetic resonance imaging identifies a subgroup of patients with asymptomatic monoclonal plasma cell disease and pathologic microcirculation. Clin Cancer Res. 2009;15(9):3118-25.

78. Dixon WT. Simple proton spectroscopic imaging. Radiology. 1984;153(1):189-94.

79. Navarro SM, Matcuk GR, Patel DB, Skalski M, White EA, Tomasian A, et al. Musculoskeletal imaging findings of hematologic malignancies. Radiographics. 2017;37(3):881-900.

80. Bauerle T, Hillengass J, Fechtner K, Zechmann CM, Grenacher L, Moehler TM, et al. Multiple myeloma and monoclonal gammopathy of undetermined significance: importance of wholebody versus spinal MR imaging. Radiology. 2009;252(2):477-85.

81. Hillengass J, Stoll J, Zechmann CM, Kunz C, Wagner B, Heiss C, et al. The application of Gadopentate-Dimeneglumin has no impact on progression free and overall survival as well as renal function in patients with monoclonal plasma cell disorders if general precautions are taken. Eur Radiol. 2015;25(3):745-50.

82. Runge VM. Critical questions regarding gadolinium deposition in the brain and Body after injections of the gadolinium-based contrast agents, safety, and clinical recommendations in consideration of the EMA's pharmacovigilance and risk assessment committee recommendation for suspension of the marketing authorizations for 4 linear agents. Investig Radiol. 2017;52(6):317-23.

83. Lancelot E. Revisiting the pharmacokinetic profiles of gadoliniumbased contrast agents: differences in long-term biodistribution and excretion. Investig Radiol. 2016;51(11):691-700.

Publisher's note Springer Nature remains neutral with regard to jurisdictional claims in published maps and institutional affiliations. 